\title{
The effect of two different visual presentation modalities on the narratives of mainstream grade 3 children
}

\author{
D Klop, PhD; L Engelbrecht, M SLT \\ Division Speech-Language and Hearing Therapy, Department of Interdisciplinary Health Sciences, Faculty of Medicine and Health Sciences, \\ Stellenbosch University, Tygerberg, Cape Town, South Africa
}

Corresponding author: D Klop (dk@sun.ac.za)

\begin{abstract}
Objective. This study investigated whether a dynamic visual presentation method (a soundless animated video presentation) would elicit better narratives than a static visual presentation method (a wordless picture book).

Method. Twenty mainstream grade 3 children were randomly assigned to two groups and assessed with one of the visual presentation methods. Narrative performance was measured in terms of micro- and macrostructure variables. Microstructure variables included productivity (total number of words, total number of T-units), syntactic complexity (mean length of T-unit) and lexical diversity measures (number of different words). Macrostructure variables included episodic structure in terms of goal-attempt-outcome (GAO) sequences. Results: Both visual presentation modalities elicited narratives of similar quantity and quality in terms of the micro- and macrostructure variables that were investigated.

Conclusion. Animation of picture stimuli did not elicit better narratives than static picture stimuli.
\end{abstract}

Keywords: digital technology, narratives, narrative elicitation procedures

S Afr J CD 2013;60:21-26 DOI:10.7196/SAJCD.242

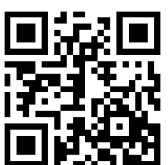

Digital technology has transformed the way we produce, distribute and receive information. Children are exposed to multiple forms of literacy including an assortment of digital literacies such as the internet, social networks (e.g. Facebook), cell phones, text messaging, instant messaging and video games, and they are also active participants in these practices. Digital technology, e.g. CD-ROMs, electronic games and electronic books, is also increasingly used in schools for learning activities such as finding information and teaching numeracy and literacy.

The availability of digital technology has changed the way speechlanguage therapists (SLTs) interact with their clients. An internet search for technological applications for use in speech-language therapy revealed a plethora of sites promising to make speechlanguage pathology easier, more fun, or just plain better' (http:// onlinespeechpathologyprograms.net/speech-sites/\#tech-res). It is observed daily in university clinics how student clinicians use their cell phones and iPads during assessment and intervention. For the past 5 years it has also been observed that students presenting seminars on the topic 'New advances in speech-language therapy', often choose topics that involve the use of technology in speech-language therapy practice. The use of digital technology may also offer more appropriate, objective and unbiased ways to assess speech-language skills. Computerised assessments may, for instance, be used to screen large groups of children for speech-language problems, making it more cost-effective than individual assessments. Clinicians who are not proficient in their clients' home languages may use computerised tests with prerecorded instructions during assessments. The use of digital assessments can also increase the reliability of assessment measures during research because the standardisation of procedures can be assured.

The goal of assessment in speech-language therapy is to obtain valid data on a child's communication performance so that appropriate goals for clinical intervention can be formulated (Owens, 2004). The assessment of children's narratives can provide valuable information not only about their speech-language skills, but also about their cognitive, pragmatic and social abilities (Liles, 1993). It is no surprise that narrative abilities are linked to academic success. The ability to tell a story links oral language skills and literacy, because it requires children to plan and produce decontextualised and cohesive narratives. Several studies (Botting, 2002; Hester, 2010; Paris \& Paris, 2003) found a positive relationship between children's reading comprehension and narrative abilities. Other studies found that narrative ability is one of the best predictors of academic success for children with language impairments (Bishop \& Edmundson, 1987; Oakhill \& Cain, 2007).

The selection of inappropriate stimuli to elicit narratives or assessment procedures that are unfamiliar to children may result in language samples that do not reflect the child's true ability and even lead to misdiagnosis. In contrast with traditional methods such as wordless picture books and sequence cards, videos and animated sequences may be more suitable to elicit narratives in South African children because they may be more familiar with information communication technologies than print texts. It can be speculated that retelling favourite televised stories may be a more common experience for them than retelling stories from other sources like storybooks. More dynamic visual modality story presentations, such as animated stories or sequences, may therefore represent more naturalistic and familiar contexts to assess their narratives.

This article reports the findings of a study that investigated whether silent animated videos elicit better narratives than a wordless picture book from typically developing grade 3 children. The quality of narratives was judged in terms of micro- and macrostructural variables. No other studies were found that compared children's narratives elicited by these two tasks using the same pictorial content. The next section will provide a brief overview of the existing literature about animation and static pictures as presentation formats and the use of visual and auditory tasks to elicit narratives for language assessment of children.

\section{Dynamic versus static picture presentation}

Static and animated pictures are both examples of a pictorial presentation format. Animation has been defined as 'any application which generates a series of frames, so that each frame appears as an alteration of the previous one, and where the sequence of frames is determined either by the designer or the user' (Bétrancourt \& Tversky, 2000 as cited in Schlosser et al., 2012, p. 342). Because animations portray temporal change, they are well suited to convey information that is inherently dynamic such as actions and movement. It seems logical that it would be easier for children to form mental models from animated sequences because the relationships between actors and scenes can be observed. In contrast, static pictures represent less realistic representations of events and require that children interpret the static events into their own dynamic representations (Gazella \& Stockman, 2003).

In a review of research about the efficacy of animated over static pictures to convey complex systems, Tversky, Morrison and 
Bétrancourt (2002) found limited evidence to support the notion that animated pictures are superior to static ones. They found that the benefits of animation over static pictures could usually be attributed to extra information or added procedures that were provided, and not the animation as such. They also cite evidence that animations may interfere with learning because they are too complex or distracting, or move too fast. More recently, studies by Stoner, Beck, Dennis and Parette (2011) and Schlosser et al. (2012) also failed to prove that animated pictures are better to facilitate vocabulary learning in preschool children than static pictures.

Of particular relevance to the present study, are the results from an intervention study by Verhallen, Bus and De Jong (2006) that investigated the effects of animated storybooks and static storybooks on children's narrative skills. Their study population comprised preschool children learning a second language. Children were exposed to stories presented on a computer screen. The static format was a voice reading a story while the pictures appeared on the screen, while the animated format was the same story supplemented by multimedia features such as animation, music and sound effects. It was found that the children exposed to the animated version obtained higher scores for story comprehension, vocabulary and syntax after repeated viewings, compared with only modest improvement in the group exposed to the static format. The researchers concluded that the additional non-verbal features in the animated format enhanced the children's awareness of implied story elements such as the causal relationships between events and mental states of the protagonists.

\section{Narrative assessment}

The assessment of narratives is considered by researchers and clinicians to be an ecologically valid way of investigating communicative competence (Botting, 2002) and to be more valid, sensitive and less biased than norm-referenced tools for assessment in some populations (Manolitsi \& Botting, 2011; Norbury \& Bishop, 2003). The inclusion of a narrative test is therefore a valuable component in any speechlanguage assessment battery.

Oral narratives also allow clinicians to analyse multiple linguistic features, such as microstructural aspects, e.g. lexical diversity, and macrostructural elements, e.g. story grammar, using a relatively short language sample (Heilmann, Miller, Nockerts \& Dunaway, 2010).

It is, however, important to be aware of the different task and modality demands when selecting stimuli and procedures to elicit narratives that are valid representations of a child's speech and language abilities (Boudreau, 2008). Research has shown that both the comprehension and production of narratives can be influenced by a number of aspects, e.g. the elicitation context (structured or unstructured), story genre (story retellings or story generation or personal stories), narrative themes, the child's experiences and world knowledge, the presentation modality (audio, visual or combined audiovisual input), and the familiarity of the listener (Gazella \& Stockman, 2003; Liles, 1993).

\section{Visual and auditory input modalities in narrative assessment}

Several researchers found that different auditory and visual presentation modes have significant effects on narrative performance. Schneider (1996) and Schneider and Dubé $(1997,2005)$ compared narratives produced in response to visual-only input, audio-only input and audiovisual input. Schneider (1996) found that 5- to 9-year-old children with language impairment produced the largest number of story grammar units in response to the audio-only condition (story retelling with no pictures) compared with the picture-only condition. Schneider and Dubé (1997) found that normally developing preschool children used references more adequately in response to an audio-only presentation than when formulating stories themselves from pictures only.

Schneider and Dubé (2005) used the same three elicitation modalities as they had previously used (1997) in similar study populations, but investigated the impact of the presentation modalities on story grammar units produced by the children. Their results indicated that all children performed similarly during the visual-only modality, and that this modality elicited the least story grammar units for both age groups. For the audio-only and combined audiovisual presentations, developmental differences were observed. Preschool children produced more story grammar units in the audiovisual condition than in the picture-only condition. Grade 2 children produced more story grammar units in both audio conditions (audio-only and audiovisual) than in the visual-only condition. They concluded that children have poorer narrative performance in visual-only tasks (pictures), because they need to apply their own internalised story knowledge to interpret the information and to produce a coherent narrative.

A few studies have also explored the use of animation in narrative assessment. Most of these studies used commercially available videos or animations that were not specifically designed and compiled for narrative elicitation (Gutierrez-Clellen \& Iglesias, 1992; Meringoff, 1980; Lorch, Berthiaume, Milich \& Van den Broek, 2007). Other studies adapted existing commercially available videos (Merritt \& Liles, 1987; Scott \& Windsor, 2000). Commercial videos may have high interest value to children, because they may be more familiar with the content of the stories. However, video stories that were not designed to contain the specific structural aspects of narratives under investigation may fail to elicit these structures in retellings. Children may fail to include certain aspects of narratives, such as settings and goals of characters, because the story did not provide enough contextual clues about these narrative aspects. This may result in lower narrative scores and stories that are not representative of children's true narrative abilities, as a result of the story content rather than the presentation modality.

\section{Wordless picture books}

In contrast to animated videos, wordless picture books have routinely been used in narrative assessment and narrative research studies. The books are usually selected because they portray the narrative structures under investigation, such as story grammar elements (i.e. settings, characters, problems, solutions). The wordless picture book Frog where are you? (Mayer, 1969) in particular, has been used by many researchers (GutierrezClellen \& Iglesias, 1992; Trabasso \& Rodkin, 1994). According to Arizpe (2013) wordless picture books are often considered by researchers the 'ideal medium' to investigate language development and storytelling skills because the books are usually brief, deal with themes that interest young readers and minimise bias against participants with lower literacy levels.

\section{The present study}

Narratives are usually analysed at two levels, namely microstructure and macrostructure. Microstructural analyses focus on the internal linguistic structures used in the construction of the narrative, such as noun phrases and conjunctions. Macrostructural analyses, by contrast, focus on higher-order hierarchical organisation such as episodic structure and story grammar components (Heilmann et al., 2010). Microstructure and macrostructure variables therefore represent two distinct areas underlying narrative competence. The variables investigated in this study were grouped into these two broad categories.

This study fell within a quantitative, comparative, between-subjects paradigm and was guided by the following research question: What is the effect of two different visual presentation modalities, an animated video and a wordless picture book, on the narrative skills of mainstream grade 3 children?

Our specific research questions were: What are the differences between the narratives of children who viewed a silent animated video and children who viewed a wordless picture book in terms of the following aspects of micro- and macrostructure:

Microstructure

- Quantity (number of words and number of T-units)?

- Lexical diversity (number of different words)?

- Syntactic complexity (mean length of T-units)?

Macrostructure

- Story structure (number of complete goal-attempt-outcome (GOA) sequences)?

Since the focus was on the effect of visual presentation, no model story or audio input was given to the participants. They were required to generate their own stories from the pictures, in other words, story generation or telling instead of retelling. Our expectation was that animation of pictures would result in longer and more complex narratives because the portrayal of movement would facilitate better understanding of the goals and intentions of characters.

A T-unit (terminal unit) comprises a main clause with all its 
concomitant subordinate clauses and phrases (Owens, 2004:190). Simple sentences, e.g. The children played outside, and complex sentences with embedded clauses e.g. Whatever she told you was true comprise one T-unit. A compound sentence where two or more clauses are conjoined, e.g. The children played outside while their mother made supper, comprises two T-units.

\section{Method}

\section{Ethical approval}

The original study from which the present study's data were extracted was approved by the institutional ethical review committee of Stellenbosch University (N09/01/016). Formal written consent was obtained from the Western Cape Education Department (2009/03200010), school principal, parents and participants prior to the study.

\section{Participants}

This study reports data of 20 children who were part of a larger project (Engelbrecht, 2010) that investigated the effect of different visual modality and task conditions on the narratives of 29 grade 3 children in a mainstream school. The initial study's participants were recruited from a primary school in the Western Cape, South Africa by sending letters to the parents of grade 3 learners in two Afrikaans-medium classes. Selection criteria specified that they should be in grade 3, Afrikaans home language speakers receiving their formal education in their home language, and that they have no speech or language problems and normal hearing. The children came from the same suburban community, cultural group and middle-class socio-economic background. A parent questionnaire was used to exclude participants with a history of speech or language problems, chronic otitis media or hearing problems. For inclusion in the study, children had to pass otoscopic examinations and pure tone hearing screening at $20 \mathrm{~dB}$ levels at 500,1 000, 2000 and 4 $000 \mathrm{~Hz}$ (Stach, 1998). A GSI 38 screening audiometer was used for the hearing screening. Children who failed the hearing screening test were excluded from the study and referred for hearing tests. Participants' receptive language skills were assessed by means of the Afrikaanse Reseptiewe Woordeskattoets (ARW) (Buitendag, 1994), a formal standardised test developed for use in the South African population. The data of 9 children who obtained ARW standard scores that were below 1 standard deviation (SD) from the average, indicative of receptive language impairments, were excluded from the study.

This article reports the results of 20 children from the original study, aged between 8 years 5 months and 9 years 4 months who complied with all the abovementioned selection criteria, and whose parents reported no history of hearing or language impairments. The original cohort $(n=29)$ was randomly assigned to either a group presented with a wordless picture book or a group presented with an animated video. The participants of this study $(n=20)$ were equally distributed between the two randomised groups. Group 1 (wordless picture book presentation) comprised 8 girls and 2 boys, and group 2 (animated video presentation) 7 girls and 3 boys. One-way analysis of variance (ANOVA) showed no differences between the groups for age, $F(1,18)=0.29, p=0.60$ and ARW standard scores, $F(1,18)=1.20, p=0.29$.

\section{Materials and instrumentation \\ Wordless picture book}

An original story, The Bird's Nest, was developed by the researchers and illustrated by a professional illustrator (see Appendix A for pictorial content). We aimed to create a story with settings (house, garden, hospital), characters (children, neighbour, doctor) and events (being bored inside the house, playing outside with a ball, getting a ball from the roof, discovering a bird's nest, getting hurt, being treated by a doctor) that would be familiar to typically developing South African children growing up in suburban areas. The picture book contained 15 colour pictures, 1 per page, and no accompanying words. The layout and printing were done by a professional company. The pictures were specifically created to convey the characters' emotions and intentions through their facial expressions and gestures. The backgrounds were kept simple so as not to distract the viewer's attention from the storyline while still providing clear settings for the events. The storyline comprised an obvious sequence of events and narrative structure.

The story events in the wordless picture book in this study were constructed to portray five possible GAO sequences. (In order for children to tell coherent narratives, they must display knowledge of the goals or plans of story characters that cause other events, attempts or outcomes in the story (Trabasso \& Rodkin, 1994). These are known as goal-attempt-outcome (GAO) sequences and are based on a shared feature of story grammar theory and the causal network model, namely a focus on characters' goals and motivations in a story and the actions and outcomes as a result of their goals (Flory et al., 2006). The content of the two visual modalities in our study was specifically designed to portray linked GAO sequences. No other studies could be found that have investigated modality presentation effects on children's production of goal-directed narratives and the inclusion of GAO sequences.) Each GAO consisted of three linked elements that were defined as follows:

Goals: Content that depicted characters' intentions, e.g. children pointing to a ball on a roof.

Attempts: Content that depicted characters' overt actions to achieve a goal, e.g. boy climbing on the roof to get the ball.

Outcomes: Content that depicted occurrences that demonstrated the results of their successful or unsuccessful attempts to reach their goals, e.g. boy falling off the roof without getting the ball.

\section{Animated video}

The animated video used the same picture sequences as the wordless picture book, but animation was added by a graphic designer to create a 2-minute video. The video was without sound, in order to simulate the wordless picture book presentation, and the pictures on the computer screen were similar in size to those in the picture book. Animation effects portrayed the movements of characters, soundless facial speaking movements and fading between pictures. A laptop computer (HP Pavilion dv5000) was used to present the animated story presentation, one picture at a time.

\section{Procedures}

Each participant was exposed to the same test conditions to increase the validity and reliability of the assessment procedures. Narratives were collected by one of the researchers, an SLT familiar with the use of narratives in assessment and intervention procedures. Assessment took place over a period of 1 week. Each child was assessed individually in a quiet room at the school. All the participants in each group were presented with the same animated video and wordless picture book by the same researcher and each session was videotaped using a Canon MV 790 video camera.

\section{Story presentation procedures}

\section{Wordless picture book}

Each participant was asked to preview the wordless picture book before telling the story. The researcher sat next to the child while the child paged through the book. The book was then closed and the child was asked to tell the story to the researcher, without looking at the pictures in the book. Participants were expected to tell the story without looking at the pictures or paging through the book again to create similar telling conditions between the wordless picture book and animated video presentation. During the story telling, the researcher responded with neutral responses such as 'hmm', 'yes' and 'what else?' to prompt the child to continue the story or to provide more information. If the child stopped the story without providing a formal ending, the researcher asked the child, 'Tell me when you have finished'.

\section{Animated video presentation}

The child viewed the animated video on a laptop computer, while the researcher sat next to the child. The child was asked to tell the story immediately after viewing the video to the researcher without looking at the video again. During the story telling, the researcher used the same neutral responses described for the wordless picture book presentation. 


\section{Data coding and analyses \\ Transcription and pruning}

All coding was done manually by the researchers. After the narratives were collected, each participant's narrative was transcribed and all revisions or self-corrections, word repetitions, interjections (e.g. 'uhm') and incomplete utterances were excluded. The remaining data were then segmented into T-units and coded for total number of words (TNW), total number of T-units (Tot T), number of different words (NDW), mean length of T-unit (MLT) and number of complete GAO sequences according to scoring protocols compiled by the researchers. A complete GAO was defined as a linked sequence of events where the narrator provides a goal statement expressing a protagonist's intention; followed by an action statement that is directly linked to the goal, i.e. an attempt to accomplish the goal; and an outcome statement, linked to the goal and the attempt, that specifies the outcome of the attempt or action, i.e. whether the goal was accomplished or not.

\section{Data analyses}

To establish inter-rater reliability, an SLT who was blinded to participants' group assignment recoded six (30\%) data sets randomly selected from all the narratives according to the coding protocol compiled by the researchers (Engelbrecht, 2010). The results of the inter-rater agreements were as follows: transcriptions 99\%; TNW 99\%; Tot T 100\%; MLT 95\%; NDW 99\%; GAOs 98\%. Differences were resolved through discussion so that consensus was reached for coding decisions.

A commercial software package, Statistica 9, was used for the statistical analyses of the data by a biostatistician. In order to examine the differences between the participants in the groups exposed to either the animation task or the wordless picture book task, one-way ANOVAs were used. A $5 \%$ significance level $(p<0.05)$ was used as a guideline for determining significant effects of variables.

\section{Results and discussion}

The main aim of this study was to determine if a dynamic visual modality in the form of an animated video presentation would elicit better narratives than a static visual modality in the form of a wordless picture book.

Table 1 provides a summary of the descriptive statistics of the participants in each group with regard to age, receptive vocabulary scores and micro- and macrostructural variables.

In summary, no significant differences between the wordless picture book and animated video modalities were found with regard to the quantity, lexical diversity and syntactic complexity measures. ANOVA showed no significant differences between the groups for TNW, $F(1,18)=0.39, p=0,54$; Tot T, $F(1,18)=0.24, p=0.62$; NDW, $F(1,18)=1.31$, $p=0.27$; MLT, $F(1,18)=0.19, p=0.67$; and GAOs, $F(1,18)=0.00, p=1.0$.

Both visual modalities elicited narratives of similar quality in terms of the microstructural variables that were investigated. These

Table 1. Means (and standard deviations) for the different variables for participants in the wordless picture book and animated video groups

\begin{tabular}{lll}
\hline & \multicolumn{2}{c}{ Participant groups } \\
\cline { 2 - 3 } Variable & $\begin{array}{l}\text { Animated video } \\
(\boldsymbol{n}=\mathbf{1 0})\end{array}$ & $\begin{array}{l}\text { Wordless picture } \\
\text { book }(\boldsymbol{n}=\mathbf{1 0})\end{array}$ \\
\hline Age (months) & $106(3.2)$ & $106(3.9)$ \\
ARW* standard scores & $100(5.7)$ & $104(8.2)$ \\
Total number of words & $162.1(42.8)$ & $174.9(47.6)$ \\
Total number of T-units & $19.5(4.3)$ & $20.5(4.6)$ \\
Mean length of T-units & $8.2(1.1)$ & $8.5(1.3)$ \\
Number of different words & $65(11.9)$ & $72(15.8)$ \\
Goal attempt outcomes & $0.8(0.8)$ & $0.8(0.9)$
\end{tabular}

*Afrikaanse Reseptiewe Woordeskattoets (Afrikaans Receptive Vocabulary Test) (Buitendag, 1994). findings concur with those of Gazella and Stockman (2003) and Schneider and Dubé (2005) who also found no differences between children's narratives when elicited by visual-only presentation modalities.

The inclusion of linked GAOs was regarded as the crucial element of macrostructural organisation in participants' narratives. A novel story portraying events that represented five potential GAOs was created by the researchers. It was hypothesised that the animation effects that portrayed movement of the characters and their facial expressions would make it easier for children to infer goals and intentions and causal connections between events. Instead we found no significant difference between the two groups with regard to the inclusion of linked GAOs. The similar performances for micro- and macrostructural measures may be because, as previously reported by Schneider and Dubé (1997), school-age children are less influenced by the elicitation modality (visual, audio or audiovisual) and require less visual support when telling stories, than preschool children. Our grade 3 participants were aged between 8 years 5 months and 9 years 4 months. It is possible that preschool children would have benefited more from a dynamic presentation mode relative to a static presentation mode and that group differences would have occurred in preschool children.

Another unexpected finding was the low frequency of GOAs in the participants' narratives. Four participants in each group, i.e. 8 of the 20 participants, included no GAOs at all in their narratives; 9 participants included only 1 GAO; 2 participants included 2 GAOs; and only 1 participant included 3 GAOs. Previous research (Stein \& Glenn, 1979, Trabasso \& Rodkin, 1994) has shown that preschool children seldom include information about the goals, intentions and internal reactions of protagonists when telling or retelling fictional narratives. The explicit inclusion of internal state terms of protagonists develop later in children's narratives and represent more advanced story grammar and narrative organisational skills (Van den Broek, Lorch \& Thurlow, 1996). However, we expected that the participants in our study, grade 3 learners aged between 8 and 10 years, would include more GOAs in their retellings, particularly in the animation group. We offer three possible explanations for the low occurrence of GAOs in participants' narratives:

First, our predetermined coding criteria for GAOs were strict in an attempt to protect the internal validity of our non-standardised protocol. Participants were only credited for the explicit statements of goals that were expressed with mental verbs such as 'want' and 'decide', goal statements that clearly denoted the intentions of protagonists, e.g. 'he climbed onto the roof to get the ball' and goal statements linked to attempt and outcome statements. Statements where goals were implied were not credited. Participants may have omitted statements of goals or intentions because they assumed that the listener could deduce these from their actions or attempt statements. As stated by Trabasso and Rodkin (1994), we cannot assume lack of goal plans or awareness of protagonists' goaldirected behaviour if a child does not explicitly link action and goal statements. The inclusion of focused questions to elicit explicit goal statements after the telling may have given us additional information about the participants' mental representations.

The second possible reason for the low inclusion of linked goal statements in both groups may be the result of the visual-only presentation modalities. We tried to portray intentions and emotions through the characters' facial expressions and gestures, but audio input such as dialogue between characters verbalising their goals and intentions may have facilitated the inclusion of more goal statements in the retell narratives.

Thirdly, the participants told their narratives to the same person who presented them with the elicitation stimuli. While this listener condition resembles the typical clinical assessment situation, researchers usually make use of a naïve listener for retellings to control for the effect of assumed shared knowledge (Gazella \& Stockman, 2003; Schneider \& Dubé, 2005). It is possible that participants would have included more explicit GAO statements to a different person acting as a naïve listener. 


\section{Study limitations}

The small sample size of this study population was a limitation. A larger sample may elicit more significant statistical differences between the visual modalities, and allow for more accurate generalisation of the results to the target population of typically developing grade 3 children. Future research on younger and language-impaired children could be beneficial in determining the developmental order of narratives in response to these two visual modalities and task conditions.

\section{Clinical implications}

The results of this study have several clinical implications for narrative assessment practices. We expected that the children would be more interested in the animated version of the stories and that the movement of the characters would elicit longer and more complex language. Participants, however, produced comparable narratives in terms of micro- and macrostructural measures in response to both visual modalities. It seems from our results that, for older typically developing children, there is no difference between the two methods. This leaves the clinician with the decision of choosing between the two modalities for narrative assessment. While the animated video may be more interesting to children, it may not be the most practical modality for clinical use. Aspects that must be kept in mind are the availability of equipment, like a television, computer or visual screen, to display the video. Therefore, in the absence of evidence that animation is superior to static elicitation stimuli the use of wordless picture books may be more practical, affordable, accessible, easily transportable, and probably less timeconsuming than videos.

The findings of this study provide information with regard to typically developing grade 3 children's production of linked GAOs in their narratives. Our results showed that $40 \%$ of the children produced no GAOs in response to pictures that portrayed a story with several episodes. They did not explicitly state every possible goal in the narrative and implied some goals by their attempt statements. This finding suggests that a narrative production measure on its own may underestimate a child's narrative abilities. The use of an additional comprehension measure with focused questions to elicit GAOs would have provided additional insight in our participants' understanding of the story. In clinical practice it may also be advisable to elicit more than one narrative, for example also a retell narrative in response to a model story, to gain a more valid representation of a child's narrative competence.

Acknowledgement. This paper is partly based on the second author's work towards a master's thesis at Stellenbosch University under the supervision of the first author. This research was supported in part by a grant from the Harry Crossley Foundation to the second author. We are grateful to the children, teacher, school and the Western Cape Education Department, whose participation and assistance made this project possible.

\section{References}

Arizpe, E. (2013). Meaning-making from wordless (or nearly wordless) picturebooks: what educational research expects and what readers have to say. Cambridge Journal of Education, doi:10.1080/0305764X.2013.767879

Bishop, D. V. M. \& Edmundson, A. (1987). Language-impaired 4-year-olds: distinguishing transient from persistent impairment. Journal of Speech and Hearing Disorders, 52, 156173.

Botting, N. (2002). Narrative as a tool for the assessment of linguistic and pragmatic impairments. Child Language Teaching and Therapy, 18(1), 1-21.

doi:10.1191\%2F0265659002ct224oa

Boudreau, D. (2008). Narrative abilities: Advances in research and implications for clinical practice. Topics in Language Disorders, 28(2), 99-114. doi:10.1097\%2F01. TLD.0000318932.08807.da

Buitendag, M. M. (1994). Afrikaanse Reseptiewe Woordeskattoets. Pretoria: Human Sciences Research Council.

Engelbrecht, L. (2010). The effect of different visual modality and task conditions on the narratives of typically developing 9 year old children. Unpublished master's thesis. Stellenbosch University, Tygerberg.
Flory, K., Milich, R., Lorch, E. P., Hayden, A. N., Strange, C. \& Welsh, R. (2006). Online story comprehension among children with ADHD: which core deficits are involved? J Abnormal Child Psychol, 34, 853-865.

Gazella, J. \& Stockman, I. J. (2003). Children's story retelling under different modality and task conditions: implications for standardizing language sampling procedures. American Journal of Speech-Language Pathology, 12, 61-72. doi:10.1044\%2F10580360\%282003\%2F053\%29

Gutierrez-Clellen, V. R. \& Iglesias, A. (1992). Causal coherence in the oral narratives of Spanish-speaking children. J Speech Lang Hear Res, 35, 363-372.

Heilmann, J., Miller, J. F., Nockerts, A. \& Dunaway, C. (2010). Properties of the narrative scoring scheme using narrative retells in young school-age children. American Journal of Speech-Language Pathology, 19, 154-166. doi:10.1044\%2F10580360\%282009\%2F08-0024\%29

Hester, E. J. (2010). Narrative correlates of reading comprehension in African-American children. Contemporary Issues in Communication Science and Disorders, 37, 73-85.

Liles, B. Z. (1993). Narrative discourse in children with language disorders and children with normal language: A critical review of the literature. J Speech Lang Hear Res, 36 868-882.

Lorch, E. P., Berthiaume, K. S., Milich, R. \& Van den Broek, P. (2007). Story comprehension impairments in children with attention-deficit/hyperactivity disorder. In K. Cain \& J. Oakhill (Eßds.), Children's comprehension problems in oral and written language (pp. 128-156). New York, NY: Guilford Press.

Manolitsi, M. \& Botting, N. (2011). Language abilities in children with autism and language impairment: using narrative as an additional source of clinical information. Child Language and Therapy, 27(1): 39-55. doi:10.1177/0265659010369991.

Mayer, M. (1969). Frog where are you? New York, NY: Dial Books for Young Readers.

Meringoff, L. (1980). A story: The influence of the medium on children's apprehension of stories. Journal of Educational Psychology, 72, 240-244.

Merritt, D. D. \& Liles, B. Z. (1987). Story grammar ability in children with and without language disorder: story generation, story retelling and story comprehension. J Speech Lang Hear Res, 30, 539-552.

Norbury, C. F. \& Bishop, D. V. M. (2003). Narrative skills of children with communication impairments. Int J Lang Commun Disord, 38, 287-313. doi:10.1080\% 2F136820310000108133

Oakhill, J. \& Cain, K. (2007). Issues of causality in children's reading comprehension. In McNamara, D. S. (Ed.). 2007. Reading comprehension strategies (pp. 47-72). New York, NY: Lawrence Erlbaum Associates.

Owens, R. E. (2004). Language disorders: A functional approach to assessment and intervention (4th ed.). Boston, MA: Allyn \& Bacon.

Paris, A. H. \& Paris, S. G. (2003). Assessing narrative comprehension in children. Reading Research Quarterly, 38(1), 36-76. doi:10.1598\%2FRRQ.38.1.3

Schlosser, R.W., Shane, H., Sorce, J., Koul, R., Bloomfield, E., Debrowski, L., DeLuca, T., Miller, S., Schneider, D. \& Neffa, A. (2012). Animation of graphic symbols representing verbs and prepositions: Effects on transparency, name agreement, and identification. J Speech Lang Hear Res, 55, 342-358. doi:10.1044/1092-4388(2011/10-0164)

Schneider, P. (1996). Effects of pictures versus orally presented stories on story retellings by children with language impairment. American Journal of Speech-Language Pathology, $5,86-96$

Schneider, P. \& Dubé, R. V. (1997). Effect of pictorial versus oral story presentation on children's use of referring expressions in retell. First Language, 5, 283-302.

Schneider, P. \& Dubé, R. V. (2005). Story presentation effects on children’s retell content. American Journal of Speech-Language Pathology, 14, 52-60. doi:10.1044\%2F10580360\% $282005 \% 2 \mathrm{~F} 007 \% 29$

Scott, C. M. \& Windsor, J. (2000). General language performance measures in spoken and written narrative and expository discourse of school-aged children with language learning disabilities. J Speech Lang Hear Res, 43, 324-339.

Stach, B. A. (1998). Clinical audiology: An introduction. London: Thomson Delmar Learning.

Stein, N. L. \& Glenn, C. G. (1979). An analysis of story comprehension in elementary schoo children. In R. O. Freedle (Ed.). New directions in discourse processing (pp. 53-119) Norwood, NJ: Ablex.

Stoner, J. B., Beck, A. R., Dennis, M. \& Parette, H. P. (2011). The use of instructional technology in direct vocabulary instruction. Journal of Special Education Technology 26(3), 35-46.

Trabasso, T. \& Rodkin, P. C. (1994). Knowledge of goal/plans: a conceptual basis for narrating Frog Where are You? In R. A. Berman \& D. I. Slobin (Eds). Relating events in narrative: A cross-linguistic developmental study (pp. 85-106). Hillsdale, NY: Lawrence Erlbaum Associates.

Tversky, B., Morrison, J. B. \& Betrancourt, M. (2002). Animation: can it facilitate? International Journal of Human-Computer Studies, 57, 247-262. doi:10.1006/ijhc.1017.

Van den Broek, P., Lorch, E. P. \& Thurlow, R. (1996). Children's and adult's memory for television stories: The role of causal factors, story-grammar categories, and hierarchical level. Child Dev, 67, 3010-3028.

Verhallen, M. J. A. J., Bus, A. G. \& de Jong, M. T. (2006). The promise of multimedia stories for kindergarten children at risk. Journal of Educational Psychology, 98(2), 410-419. doi:10.1037/0022-0663.98.2 

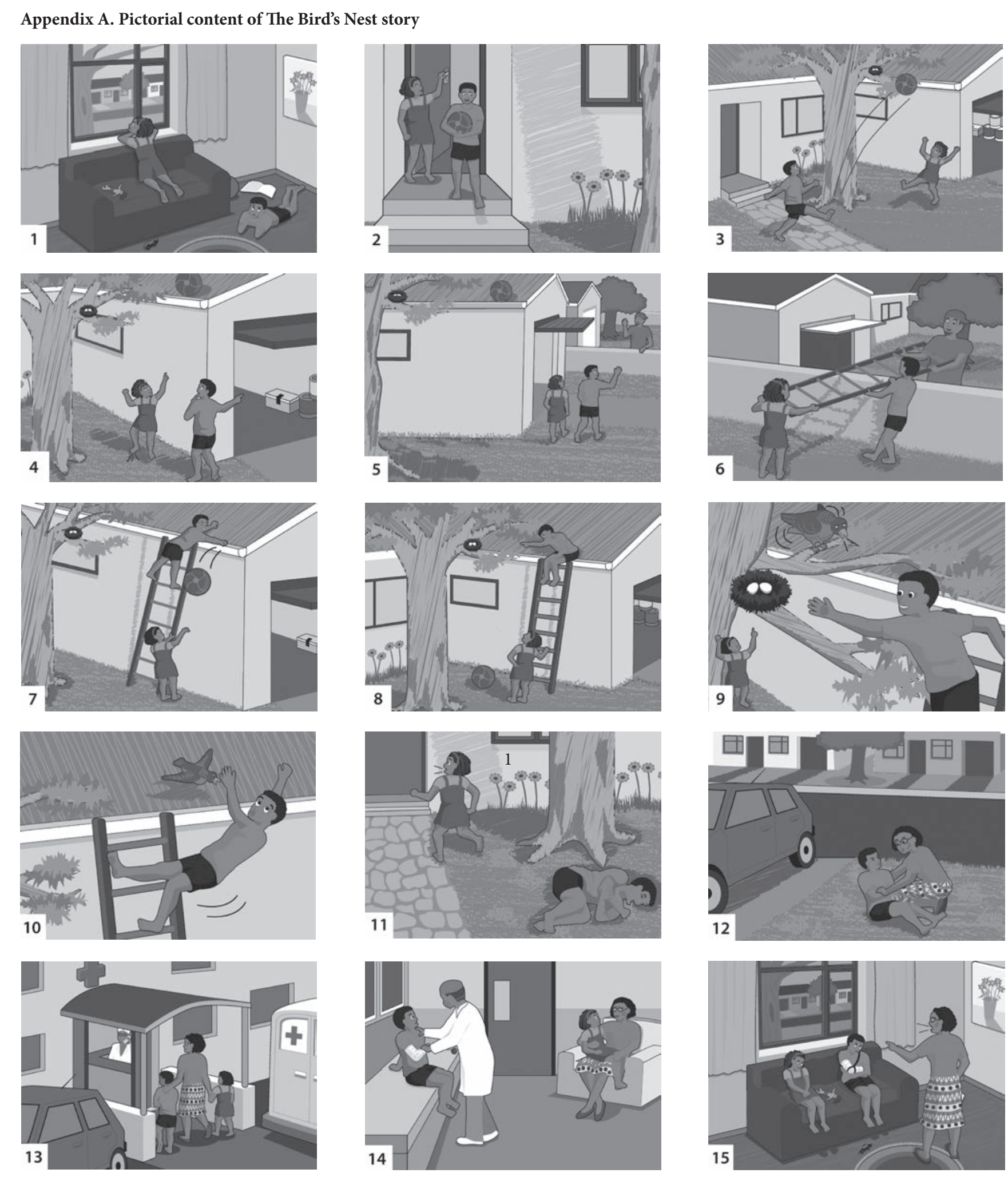